\title{
Improving the stability of a simple formulation of the shallow water equations for 2-D flood modeling
}

\author{
Gustavo A. M. de Almeida, ${ }^{1}$ Paul Bates, ${ }^{1}$ Jim E. Freer, ${ }^{1}$ and Maxime Souvignet ${ }^{1}$ \\ Received 31 October 2011; revised 27 March 2012; accepted 28 March 2012; published 17 May 2012.
}

[1] The ability of two-dimensional hydrodynamic models to accurately and efficiently predict the propagation of floods over large urban areas is of paramount importance for flood risk assessment and management. Paradoxically, it is in these highly relevant urban domains where flood modeling faces some of the most challenging obstacles. This is because of the very high-resolution topography that is typically required to capture key hydraulic features, which significantly increases the computational time of the model. One particularly interesting solution to this difficulty was recently proposed in the form of a numerical scheme for the solution of a simplified version of the shallow water equations, which yields a system of two explicit equations that captures the most relevant hydraulic processes at very high computational efficiency. However, some stability problems were reported, especially when this formulation is applied to low friction areas. This is of particular importance in urban areas, where smooth surfaces are usually abundant. This paper proposes and tests two modifications of this previous numerical scheme that considerably improves the numerical stability of the model. Model improvements were assessed against a structured set of idealized test cases and finally in the simulation of flood propagation over complex topography in a highly urbanized area in London, United Kingdom. The enhanced stability achieved by the new formulation comes at no significant additional computational cost and, in fact, the model performance can benefit from the longer time steps that are allowed by the new scheme.

Citation: de Almeida, G. A. M., P. Bates, J. E. Freer, and M. Souvignet (2012), Improving the stability of a simple formulation of the shallow water equations for 2-D flood modeling, Water Resour. Res., 48, W05528, doi:10.1029/2011WR011570.

\section{Introduction}

[2] Two-dimensional flood inundation modeling is a pivotal component of flood risk assessment and management. It is therefore not surprising that over the last decades significant efforts have been devoted to the development of increasingly complex algorithms to simulate the flow of water in streams and floodplains [e.g., Peraire et al., 1986; Bermudez et al., 1991; Hervouet and Petitjean, 1999; Hervouet, 2000; Toro, 2001; Guinot and Frazão, 2006; Toro and Garcia-Navarro, 2007; LeVeque and George, 2008; Sanders et al., 2008; Li and Duffy, 2011].

[3] With the growing availability of satellite and airborne terrain data, simulations at very high resolution have become possible. This is particularly important in urban areas, where a detailed representation of complex topographical features is crucial to correctly reproduce key hydrodynamic processes [e.g., Horritt and Bates, 2001; Brown et al., 2007].

[4] However, the extremely high computational cost of simulations using sophisticated models over large areas and at high resolution still imposes substantial limitations for flood modeling in many urban areas. This restriction

${ }^{1}$ School of Geographical Sciences, University of Bristol, Bristol, UK.

Copyright 2012 by the American Geophysical Union 0043-1397/12/2011WR011570 has paved the way for the development of simplified formulations that capture the most relevant mechanisms of flood propagation with very high computational performance [e.g., Ponce et al., 1978; Ponce, 1990; Xia, 1994; Aronica et al., 1998; Arico et al., 2011]. The reduction in computational time achieved by these models prompts a whole range of new applications of hydraulic models [Fewtrell et al., 2008].

[5] One simplified formulation of particular interest for flood modeling is that obtained by neglecting only the convective acceleration term in the Saint-Venant momentum conservation equation. The resulting system of partial differential equations (PDEs), variously referred to as "local inertial," "simplified inertial," or simply "inertial," has been used and analyzed in a number of previous studies [e.g., Ponce, 1990; Xia, 1994; Aronica et al., 1998; Bates et al., 2010]. Compared with other simplified versions of the Saint-Venant equations that neglect both local and convective acceleration terms (i.e., the diffusive formulation), one of the main advantages of the local inertial equations is related to the stability condition in explicit finite difference schemes. Namely, the maximum stable time step in the explicit diffusive model decreases quadratically with grid refinements [Hunter et al., 2006], while a linear relation is obtained in the case of numerical schemes based on the hyperbolic local inertial system. This leads to a considerable gain in computational performance at fine resolution, 
where the explicit diffusive approach becomes unfeasibly slow [Bates et al., 2010].

[6] Bates et al. [2010] proposed a numerical scheme for the solution of the local inertial system in which water flows through each face of regular grid computational cells are estimated by solving the inertial momentum equation using a single explicit finite difference formulation. The continuity equation is then applied to the 2-D grid domain to update water depths inside closed computational cells. The method was developed from conception to directly make use of the expanding wealth of raster terrain data now available, and therefore is only applicable to structured grids. The resulting model is relatively simple, yet contains sufficient shallow water physics to describe gradually varying unsteady flood flows adequately while requiring approximately an order of magnitude fewer numerical operations to solve than a full-dynamic shallow water model [Neal et al., 2011a]. However, some stability problems remain unsolved. Namely, Bates et al. [2010] reported that considerable numerical instabilities arise at low friction. These instabilities represent an important obstacle to the application of the model in urban areas, where relatively smooth surfaces are typically found.

[7] In this paper we propose a modification of the numerical scheme used by Bates et al. [2010] to solve the system of hyperbolic PDEs that considerably improves the model stability. After a brief mathematical analysis of the simplified mathematical model and the corresponding numerical method proposed by Bates et al. [2010], two new numerical schemes are proposed and then tested in idealized scenarios of increasing difficulty in terms of numerical stability and accuracy. Results show significant improvements in the model stability at low friction for a very small increase in computational cost that is more than offset by the longer time steps allowed as a result of the more stable scheme. The model is finally tested in a real-world simulation of a highly urbanized area in London, UK.

\section{Mathematical Framework}

[8] The starting point for the derivation of the mathematical formulation used in the model is the full-dynamic version of the 1-D Saint-Venant equations [Cunge et al., 1980]:

$$
\begin{gathered}
\frac{\partial A}{\partial t}+\frac{\partial Q}{\partial x}=0 \\
\underbrace{\frac{\partial Q}{\partial t}}_{\begin{array}{c}
\text { local } \\
\text { acceleration }
\end{array}}+\underbrace{\frac{\partial}{\partial x}\left(\frac{Q^{2}}{A}\right)}_{\begin{array}{c}
\text { convective } \\
\text { acceleration }
\end{array}}+\underbrace{g A \frac{\partial(h+z)}{\partial x}}_{\begin{array}{c}
\text { pressure } \\
\text { bed gradients }
\end{array}}+\underbrace{\frac{g n^{2}|Q| Q}{R^{4 / 3} A}}_{\text {friction }}=0
\end{gathered}
$$

where $h[L]$ is the water depth, $Q\left[L^{3} T^{-1}\right]$ is the discharge, $z[L]$ is the bed elevation, $A\left[L^{2}\right]$ is the flow cross section area, $R[L]$ is the hydraulic radius, $g\left[L T^{-2}\right]$ is the acceleration due to gravity, $n\left[T L^{-1 / 3}\right]$ is the Manning friction coefficient, $x[L]$ is the longitudinal coordinate, and $t[T]$ is the time.

[9] By neglecting the convective acceleration term in (2), dividing by the width $b$, and assuming $R=h$ (i.e., neglecting lateral friction), the following simplified inertial system is obtained:

$$
\begin{gathered}
\frac{\partial h}{\partial t}+\frac{\partial q}{\partial x}=0 \\
\frac{\partial q}{\partial t}+g h \frac{\partial(h+z)}{\partial x}+\frac{g n^{2}|q| q}{h^{7 / 3}}=0
\end{gathered}
$$

where $q\left[L^{2} T^{-1}\right]$ is the discharge per unit width.

[10] In the 2-D model proposed by Bates et al. [2010] this simplified inertial version of the 1-D momentum conservation equation (i.e., equation (4)) is used to estimate the flow between cells, while the 2-D version of the continuity equation is used to couple $x$ and $y$ directions, yielding the following system of three partial differential equations:

$$
\begin{gathered}
\frac{\partial h}{\partial t}+\frac{\partial q_{x}}{\partial x}+\frac{\partial q_{y}}{\partial y}=0, \\
\frac{\partial q_{x}}{\partial t}+g h \frac{\partial(h+z)}{\partial x}+\frac{g n^{2}\left|q_{x}\right| q_{x}}{h^{7 / 3}}=0, \\
\frac{\partial q_{y}}{\partial t}+g h \frac{\partial(h+z)}{\partial y}+\frac{g n^{2}\left|q_{y}\right| q_{y}}{h^{7 / 3}}=0 .
\end{gathered}
$$

[11] In order to study the behavior of these equations under low friction conditions we first rewrite the system (3) and (4) in terms of $u$ (flow velocity $u=q / h$ ) and $h$, and then in homogeneous, vectorial form by completely neglecting friction and assuming a flat bed:

$$
(W)_{t}+F(W)_{x}=0
$$

where $W$ is the vector of conserved variables $W=[h h u]^{T}$, $F(W)$ is the flux vector,

$$
F(W)=\left[\begin{array}{c}
u h \\
\frac{1}{2} g h^{2}
\end{array}\right],
$$

and subscripts $t$ and $x$ in (8) are used to denote the partial derivatives of $W$ with respect to $t$ and $x$, respectively. This system can now be written in quasi-linear form as,

$$
(W)_{t}+F^{\prime} W_{x}=0
$$

where $F^{\prime}(W)$ is the Jacobian matrix

$$
F^{\prime}(W)=\left[\begin{array}{ll}
\frac{\partial F_{1}}{\partial W_{1}} & \frac{\partial F_{2}}{\partial W_{2}} \\
\frac{\partial F_{2}}{\partial W_{1}} & \frac{\partial F_{1}}{\partial W_{2}}
\end{array}\right]=\left[\begin{array}{ll}
0 & 1 \\
g h & 0
\end{array}\right] .
$$

[12] The eigenvalues of this Jacobian matrix are,

$$
\begin{aligned}
& \lambda^{1}=-\sqrt{g h}, \\
& \lambda^{2}=+\sqrt{g h},
\end{aligned}
$$


and the corresponding right eigenvectors $r^{1}=[1-\sqrt{g h}]^{T}$ and $r^{2}=[1+\sqrt{g h}]^{T}$.

[13] The system is thus nonlinear and strictly hyperbolic as long as the flow depth is nonzero; and it is well known that no smooth solution will, in general, exist for all time because of this nonlinearity [e.g., Lax and Wendroff, 1960; LeVeque, 2002]. The structure of the solutions of this system is relatively simple. When the water depth is higher on the left than on the right, a shock wave will propagate to the right while a rarefaction wave propagates in the left direction. Conversely, when the depth on the right is highest, the solution will contain a left-going shock and rightgoing rarefaction. In either case a shock will develop, posing a significant challenge to the numerical method. Shock waves are discontinuities in the solution of the homogeneous (i.e., frictionless) hyperbolic system, which, in general, cannot be captured by simple finite difference methods. This is because the self-formed discontinuities will lead to the local overshooting of the spatial derivatives approximated with the finite difference schemes, which rapidly propagate to neighboring cells.

[14] As friction is reduced the system of equations (3) and (4) tends to the homogeneous equations (i.e., equation (8)) in which discontinuities are expected to develop. This paves the way to the emergence of unphysical spurious oscillations in the numerical solution, such as those reported by Bates et al. [2010].

[15] A relatively widespread solution to this type of problem is the use of shock-capturing schemes (e.g., highresolution Godunov-type finite volume methods) that explicitly resolve discontinuities by solving the local Riemann problem at the interface of two computing cells [e.g., van Leer, 1979; LeVeque, 2002; Toro, 2001; Bradford and Sanders, 2002, 2005]. This family of methods is particularly interesting for flows with physical discontinuities, such as the initial condition of a dam break problem. The computational cost of these high-resolution shock-capturing methods is, however, usually higher than simple finite difference schemes typically used in flood modeling [Neal et al., 2011a].

[16] Most flows over floodplains and in streams display relatively smooth spatial variations in the water surface, and even though steep localized changes may occur at shallow zones of the domain, they usually span over finite lengths of a few computational grid cells. The emergence of numerical discontinuities in the solution when modeling this physical situation is a result of the inherently simplified character of the mathematical representation of reality. In the real world, a gradual steepening of, e.g., the water profile would be counterbalanced by other effects such as vertical velocities (which are neglected in the shallow-water formulation) or turbulence. Modeling these additional mechanisms would require the inclusion of higher-order terms in the equations and as a result, the equations would exhibit smooth solutions [LeVeque, 2002]. Accordingly, although capturing shocks as sharp discontinuities in the solution represents a better approximation of the mathematical equations, it does not necessarily represent the physical reality better than simplified numerical approaches that numerically diffuse these discontinuities (especially in physical situations where smooth solutions are expected). In this paper we take this pragmatic point of view and propose a numerically diffusive scheme that considerably improves the stability of the model with no significant additional computational cost. Special problems, where the accurate representation of extremely steep wave fronts are needed, require more sophisticated numerical schemes, such as those mentioned in the previous paragraph.

\section{Numerical Solution}

\subsection{Numerical Scheme of Bates et al. [2010]}

[17] The numerical solution proposed by Bates et al. [2010] is implemented using a simple finite difference scheme applied to a staggered grid. In this scheme the continuity equation is solved with a Godunov-like method on square cells (Figure 1), where mass fluxes between cells are used to update water depths inside the cells. The scheme thus ensures mass conservation (i.e., cell outflows are always equal to the inflow of a neighboring cell), which constitutes an important feature for flood modeling. Unlike the Godunov method (where both mass and momentum fluxes are calculated by solving the local Riemann problem), the mass flux (i.e., flow discharge) is obtained by solving the 1-D simplified momentum equation at interfaces between cells using the value of $q$ at these interfaces (rather than in the center of cells). Furthermore, momentum itself is updated at these interfaces with an explicit discretization of the momentum equation (also unlike finite volume methods where momentum fluxes are calculated and used to update momentum inside closed cells). Finally, an adaptive time stepping method is used to estimate the suitable model time step based on the standard Courant-Friedrichs-Lewy (CFL) condition:

$$
C_{r}=\frac{\lambda \Delta t}{\Delta x}
$$

where the dimensionless Courant number $C_{r}$ needs to be less than 1 for stability and $\lambda=\sqrt{g h}$ is the wave celerity. Equation (14) gives a necessary but not sufficient condition

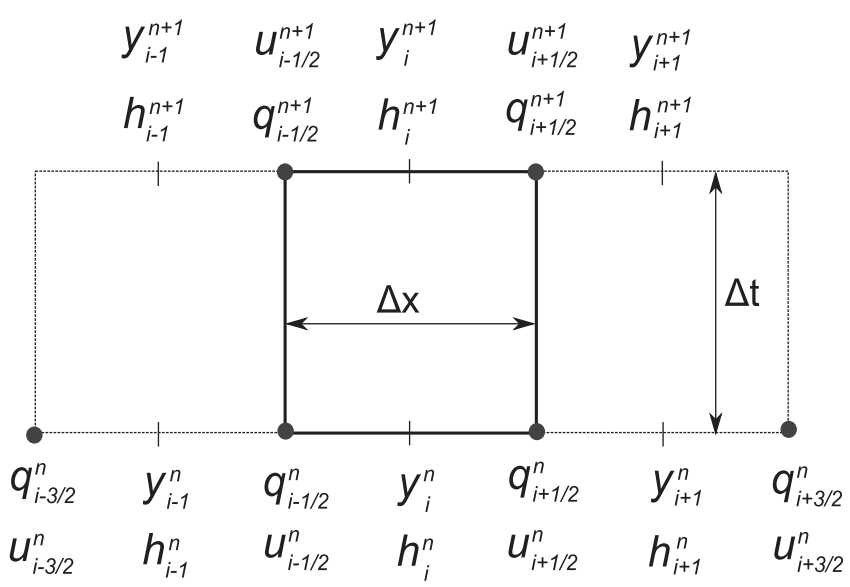

Figure 1. Grid and variables used in the numerical scheme. Only the $x$ spatial dimension is shown here for simplicity. Note the staggered characteristic of the grid: $y$ (or alternatively, $h$ ) variables are evaluated in the center of cells, while $q$ (or alternatively $u$ ) variables are evaluated at cells interfaces. 
for model stability, and the model estimates the time step as,

$$
\Delta t=\alpha \frac{\Delta x}{\sqrt{g h_{\max }}},
$$

where $h_{\max }$ is the maximum depth within the computational domain. The current version of the model uses a default value of $\alpha=0.7$, although this can be tuned by the user.

[18] The resulting numerical method is relatively simple and extremely efficient from the computational point of view, representing an excellent compromise between accuracy and performance for simulating gradually varied in time, subcritical floodplain flows.

[19] In this section we analyze the Bates et al. [2010] numerical method in terms of its stability for low friction simulations. For simplicity, the following discussion will focus on the 1-D version of the equations (i.e., equations (3) and (4)). However, the conclusions obtained for 1-D are directly transferable to 2-D as a result of the uncoupled nature of the proposed equations.

[20] The continuity equation is discretized using the following approximations for the space and time partial derivatives at the cell center:

$$
\begin{gathered}
\left.\frac{\partial y}{\partial t}\right|_{i}=\frac{y_{i}^{n+1}-y_{i}^{n}}{\Delta t}, \\
\left.\frac{\partial q}{\partial x}\right|_{i}=\frac{q_{i+1 / 2}^{n+1}-q_{i-1 / 2}^{n+1}}{\Delta x},
\end{gathered}
$$

where $y=h+z$ is the water surface elevation.

[21] Similar forward in time and centered in space difference formulae are used to discretize the momentum equation, but in this case the derivatives are approximated at the cell interfaces rather than at the cell center:

$$
\begin{gathered}
\left.\frac{\partial q}{\partial t}\right|_{i-1 / 2}=\frac{q_{i-1 / 2}^{n+1}-q_{i-1 / 2}^{n}}{\Delta t} \\
\left.\frac{\partial y}{\partial x}\right|_{i-1 / 2}=\frac{y_{i}^{n}-y_{i-1}^{n}}{\Delta x} .
\end{gathered}
$$

[22] Note that the space derivatives are estimated at $n+1$ and $n$ time steps for the continuity and momentum equations, respectively. Substituting these into equations (3) and (4), the following explicit finite difference formulae are obtained:

$$
\begin{gathered}
y_{i}^{n+1}=y_{i}^{n}+\frac{\Delta t}{\Delta x}\left(q_{i-1 / 2}^{n+1}-q_{i+1 / 2}^{n+1}\right), \\
q_{i-1 / 2}^{n+1}=q_{i-1 / 2}^{n}-g h_{f}^{n} \Delta t\left[\frac{\left(y_{i}^{n}-y_{i-1}^{n}\right)}{\Delta x}+\frac{n^{2}\left|q_{i-1 / 2}^{n}\right| q_{i-1 / 2}^{n}}{h_{f}^{10 / 3}}\right],
\end{gathered}
$$

where subscripts $i$ and $n$ denote space and time indices, respectively (Figure 1). Here the nonlinear coefficient $h_{f}$ is calculated as the difference between $\max \left(y_{i}, y_{i-1}\right)$ and $\max \left(z_{i}, z_{i-1}\right)$.
[23] To improve the stability, equation (21) was further modified to include $q_{i-1 / 2}^{n+1}$ in the estimation of the friction term, resulting in the following scheme:

$$
q_{i-1 / 2}^{n+1}=q_{i-1 / 2}^{n}-g h_{f} \Delta t\left[\frac{\left(y_{i}^{n}-y_{i-1}^{n}\right)}{\Delta x}+\frac{n^{2}\left|q_{i-1 / 2}^{n}\right| q_{i-1 / 2}^{n+1}}{h_{f}^{10 / 3}}\right] .
$$

[24] This scheme will hereafter be referred to as "semiimplicit," as it includes a $q_{i-1 / 2}^{n+1}$ term on both sides of (22), even though this equation can be rearranged to yield an explicit formulation:

$$
q_{i-1 / 2}^{n+1}=\frac{q_{i-1 / 2}^{n}-g h_{f} \frac{\Delta t}{\Delta x}\left(y_{i}^{n}-y_{i-1}^{n}\right)}{1+g \Delta t n^{2}\left|q_{i-1 / 2}^{n}\right| / h_{f}^{7 / 3}} .
$$

[25] In order to gain insight into the behavior of these equations we derive the modified equations by substituting a Taylor series expansion into the discrete system (equations (20) and (22)). This technique is illuminating, as it is usually easier to analyze the behavior of differential equations than that of their finite difference counterparts. To this end we simplify the equations by assuming a frictionless bed, and linearize the system by freezing the nonlinear coefficients (thus assuming that only small perturbations around a constant state propagate in the solution), yielding:

$$
\begin{gathered}
\frac{\partial y}{\partial t}+\frac{\partial q}{\partial x}=0, \\
\frac{\partial q}{\partial t}+g h_{f} \frac{\partial y}{\partial x}=0,
\end{gathered}
$$

where $h_{f}$ is a constant in (25).

[26] Substituting the Taylor series expansions about $i$ :

$$
\begin{aligned}
& q_{i-1 / 2}^{n+1}= q_{i}^{n+1}-\left.\frac{\partial q}{\partial x}\right|_{i} ^{n+1} \frac{\Delta x}{2}+\left.\frac{1}{2} \frac{\partial^{2} q}{\partial x^{2}}\right|_{i} ^{n+1} \frac{\Delta x^{2}}{4} \\
&-\left.\frac{1}{6} \frac{\partial^{3} q}{\partial x^{3}}\right|_{i} ^{n+1} \frac{\Delta x^{3}}{8}+\mathcal{O}\left(\Delta x^{4}\right) \\
& q_{i+1 / 2}^{n+1}= q_{i}^{n+1}+\left.\frac{\partial q}{\partial x}\right|_{i} ^{n+1} \frac{\Delta x}{2}+\left.\frac{1}{2} \frac{\partial^{2} q}{\partial x^{2}}\right|_{i} ^{n+1} \frac{\Delta x^{2}}{4} \\
&+\left.\frac{1}{6} \frac{\partial^{3} q}{\partial x^{3}}\right|_{i} ^{n+1} \frac{\Delta x^{3}}{8}+\mathcal{O}\left(\Delta x^{4}\right) \\
& y_{i}^{n+1}=y_{i}^{n}+\left.\frac{\partial y}{\partial t}\right|_{i} ^{n} \Delta t+\left.\frac{1}{2} \frac{\partial^{2} y}{\partial t^{2}}\right|_{i} ^{n} \Delta t^{2}+\left.\frac{1}{6} \frac{\partial^{3} y}{\partial t^{3}}\right|_{i} ^{n} \Delta t^{3}+\mathcal{O}\left(\Delta t^{4}\right)
\end{aligned}
$$

into equation (20), and about $i-1 / 2$ :

$$
\begin{aligned}
q_{i-1 / 2}^{n+1}= & q_{i-1 / 2}^{n}+\left.\frac{\partial q}{\partial t}\right|_{i-1 / 2} ^{n} \Delta t+\left.\frac{1}{2} \frac{\partial^{2} q}{\partial t^{2}}\right|_{i-1 / 2} ^{n} \Delta t^{2} \\
& +\left.\frac{1}{6} \frac{\partial^{3} q}{\partial t^{3}}\right|_{i-1 / 2} ^{n} \Delta t^{3}+\mathcal{O}\left(\Delta t^{4}\right)
\end{aligned}
$$




$$
\begin{aligned}
y_{i}^{n}= & y_{i-1 / 2}^{n}+\left.\frac{\partial y}{\partial x}\right|_{i-1 / 2} ^{n} \frac{\Delta x}{2}+\left.\frac{1}{2} \frac{\partial^{2} y}{\partial x^{2}}\right|_{i-1 / 2} ^{n} \frac{\Delta x^{2}}{4} \\
& +\left.\frac{1}{6} \frac{\partial^{3} y}{\partial x^{3}}\right|_{i-1 / 2} ^{n} \frac{\Delta x^{3}}{8}+\mathcal{O}\left(\Delta x^{4}\right), \\
y_{i-1}^{n}= & y_{i-1 / 2}^{n}-\left.\frac{\partial y}{\partial x}\right|_{i-1 / 2} ^{n} \frac{\Delta x}{2}+\left.\frac{1}{2} \frac{\partial^{2} y}{\partial x^{2}}\right|_{i-1 / 2} ^{n} \frac{\Delta x^{2}}{4} \\
& -\left.\frac{1}{6} \frac{\partial^{3} y}{\partial x^{3}}\right|_{i-1 / 2} ^{n} \frac{\Delta x^{3}}{8}+\mathcal{O}\left(\Delta x^{4}\right)
\end{aligned}
$$

into equation (22) (ignoring the friction term), and neglecting terms of order higher than 2, the following system of modified equations is obtained:

$$
\begin{gathered}
\frac{\partial y}{\partial t}+\frac{\partial q}{\partial x}=-\left(\frac{1}{2} \frac{\partial^{2} y}{\partial t^{2}} \Delta t+\frac{1}{6} \frac{\partial^{3} y}{\partial t^{3}} \Delta t^{2}+\frac{1}{24} \frac{\partial^{3} q}{\partial x^{3}} \Delta x^{2}\right) \\
\frac{\partial q}{\partial t}+g h_{f} \frac{\partial y}{\partial x}=-\left(\frac{1}{2} \frac{\partial^{2} q}{\partial t^{2}} \Delta t+\frac{1}{6} \frac{\partial^{3} q}{\partial t^{3}} \Delta t^{2}+g h_{f} \frac{1}{24} \frac{\partial^{3} y}{\partial x^{3}} \Delta x^{2}\right)
\end{gathered}
$$

[27] In these equations the left-hand side (LHS) is identical to that of equations (24) and (25), while the right-and side (RHS) represents the error terms resulting from the finite difference approximations introduced to discretize the system. We observe that all second order spatial derivatives in the Taylor series expansions were canceled out so that the error terms exhibit no diffusive terms. Therefore, discontinuities forming in the solution as a result of the nonlinearity will not be numerically diffused by the semi-implicit scheme, eventually inducing the instability problems reported by Bates et al. [2010]. Now, considering the complete momentum equation (i.e., including friction), it is clear that when the Manning's $n$ is relatively high, these discontinuities are damped, as high-velocity points are decelerated by the action of friction. This often masks the unstable character of the forward in time-centered in space (FTCS) scheme. In order to improve the stability of the model, in sections $3.2,3.3$, and 4 we propose and test two modifications of this numerical scheme which introduce diffusive terms to the method.

\section{2. q-Upwind Numerical Scheme}

[28] The first scheme proposed here is inspired by the notion of upwinding, but uses the flow direction rather than the direction of propagation of individual waves in an uncoupled system. The scheme is not, strictly speaking, "upwind," although this term is used here because of its similarity with the concept of upwinding. The objective of this method is to modify the finite difference approximation of $\partial q / \partial t$ by using a one-sided scheme to introduce a numerical diffusive term in terms of $q$ to the method. The time derivative of $q$ is estimated at the cell interface $i-1 / 2$ using a weighted average of $q_{i-1 / 2}^{n}$ and the upwind value of $q$ :

$$
\left.\frac{\partial q}{\partial t}\right|_{i-1 / 2}=\frac{q_{i-1 / 2}^{n+1}-\left[\theta q_{i-1 / 2}^{n}+(1-\theta) q_{u p w}^{n}\right]}{\Delta t}
$$

where $\theta$ is the weighting factor and $q_{u p w}$ takes the values of $q^{n}$ at the upstream cell interface. Therefore, if $q_{i-1 / 2}^{n}>0$, the momentum equation is discretized as,

$$
\begin{aligned}
q_{i-1 / 2}^{n+1}= & \left.\theta \theta q_{i-1 / 2}^{n}+(1-\theta) q_{i-3 / 2}^{n}\right] \\
& -g h_{f} \Delta t\left[\frac{\left(y_{i}^{n}-y_{i-1}^{n}\right)}{\Delta x}+\frac{n^{2}\left|q_{i-1 / 2}^{n}\right| q_{i-1 / 2}^{n+1}}{h_{f}^{10 / 3}}\right],
\end{aligned}
$$

and if $q_{i-1 / 2}^{n}<0$ :

$$
\begin{aligned}
q_{i-1 / 2}^{n+1}= & {\left[\theta q_{i-1 / 2}^{n}+(1-\theta) q_{i+1 / 2}^{n}\right] } \\
& -g h_{f} \Delta t\left[\frac{\left(y_{i}^{n}-y_{i-1}^{n}\right)}{\Delta x}+\frac{n^{2}\left|q_{i-1 / 2}^{n}\right| q_{i-1 / 2}^{n+1}}{h_{f}^{10 / 3}}\right] .
\end{aligned}
$$

[29] As in section 3.1, in order to analyze the behavior of this scheme we derive the modified momentum equation (the corresponding continuity equation is unchanged) by substituting equations (29)-(31) and

$$
\begin{aligned}
q_{i-3 / 2}^{n+1}= & q_{i-1 / 2}^{n}-\left.\frac{\partial q}{\partial x}\right|_{i-1 / 2} ^{n} \Delta x+\left.\frac{1}{2} \frac{\partial^{2} q}{\partial x^{2}}\right|_{i-1 / 2} ^{n} \Delta x^{2} \\
& -\left.\frac{1}{6} \frac{\partial^{3} q}{\partial x^{3}}\right|_{i-1 / 2} ^{n} \Delta x^{3}+\mathcal{O}\left(\Delta t^{4}\right)
\end{aligned}
$$

into (35) (also neglecting the friction term), which yields:

$$
\begin{aligned}
\frac{\partial q}{\partial t}+g h_{f} \frac{\partial y}{\partial x}= & -\frac{1}{2} \frac{\partial^{2} q}{\partial t^{2}} \Delta t-\frac{1}{6} \frac{\partial^{3} q}{\partial t^{3}} \Delta t^{2} \\
& +(1-\theta) \frac{\Delta x}{\Delta t}\left[-\frac{\partial q}{\partial x}+\frac{1}{2} \frac{\partial^{2} q}{\partial x^{2}} \Delta x-\frac{1}{6} \frac{\partial^{3} q}{\partial x^{3}} \Delta x^{2}\right] \\
& -g h_{f} \frac{1}{24} \frac{\partial^{3} y}{\partial x^{3}} \Delta x^{2}
\end{aligned}
$$

[30] We now observe that this scheme introduces a diffusive term in terms of the $q$ variable (i.e., the fourth term on the RHS of equation (38)), which helps to stabilize the solution. Furthermore, the amount of diffusion can be controlled by selecting suitable values of $\theta$. In particular, if $\theta=1$, then the semi-implicit scheme of Bates et al. [2010] (i.e., simple forward in time finite difference) is obtained. On the other hand, the method also adds an unwanted first order derivative term (third term on the RHS) which does not vanish with grid refinements (as $\Delta x / \Delta t$ can be assumed a constant which is constrained by the CourantFriedrichs-Lewy condition).

\section{3. q-centered Numerical Scheme}

[31] The second scheme is very similar to the q-upwind scheme, but uses information on both neighboring cell interfaces instead of the upwind value alone,

$$
\left.\frac{\partial q}{\partial t}\right|_{i-1 / 2}=\frac{q_{i-1 / 2}^{n+1}-\left[\theta q_{i-1 / 2}^{n}+\frac{(1-\theta)}{2}\left(q_{i-3 / 2}^{n}+q_{i+1 / 2}^{n}\right)\right]}{\Delta t} .
$$

[32] This is similar to the approximation of the time derivative used by the Lax diffusive scheme, but here three 
points are used to estimate $q_{i-1 / 2}^{n}$ instead of two. In fact, $q_{i-1 / 2}^{n}$ is estimated as a weighted average of $q_{i-1 / 2}^{n}$ and $q^{n}$ values in the two neighboring cell interfaces. If $\theta=0$, then the Lax approximation is obtained, while $\theta=1$ restores the semi-implicit formulation (as in the case of the q-upwind scheme).

[33] Substituting this approximation into (4) and writing the friction term as in the work of Bates et al. [2010] results in:

$$
\begin{aligned}
q_{i-1 / 2}^{n+1}= & {\left[\theta q_{i-1 / 2}^{n}+\frac{(1-\theta)}{2}\left(q_{i-3 / 2}^{n}+q_{i+1 / 2}^{n}\right)\right] } \\
& -g h_{f} \Delta t\left[\frac{\left(y_{i}^{n}-y_{i-1}^{n}\right)}{\Delta x}+\frac{n^{2}\left|q_{i-1 / 2}^{n}\right| q_{i-1 / 2}^{n+1}}{h_{f}^{10 / 3}}\right],
\end{aligned}
$$

which can be easily rearranged to yield an explicit formula:

$$
q_{i-1 / 2}^{n+1}=\frac{\left[\theta q_{i-1 / 2}^{n}+\frac{(1-\theta)}{2}\left(q_{i-3 / 2}^{n}+q_{i+1 / 2}^{n}\right)\right]-g h_{f} \frac{\Delta t}{\Delta x}\left(y_{i}^{n}-y_{i-1}^{n}\right)}{1+g \Delta t n^{2}\left|q_{i-1 / 2}^{n}\right| / h_{f}^{7 / 3}} .
$$

[34] The modified equation for this numerical scheme is again obtained by substituting the Taylor series expansions about $i-1 / 2$, i.e., equations (29), (30), (31), (37), and

$$
\begin{aligned}
q_{i+1 / 2}^{n}= & q_{i-1 / 2}^{n}+\left.\frac{\partial q}{\partial x}\right|_{i-1 / 2} ^{n} \Delta x+\left.\frac{1}{2} \frac{\partial^{2} q}{\partial x^{2}}\right|_{i-1 / 2} ^{n} \Delta x^{2} \\
& +\left.\frac{1}{6} \frac{\partial^{3} q}{\partial x^{3}}\right|_{i-1 / 2} ^{n} \Delta x^{3}+\mathcal{O}\left(\Delta t^{4}\right),
\end{aligned}
$$

into equation (40) (again ignoring the friction term). After neglecting terms of order higher than 2 this results in:

$$
\begin{aligned}
\frac{\partial q}{\partial t}+g h_{f} \frac{\partial y}{\partial x}= & -\frac{1}{2} \frac{\partial^{2} q}{\partial t^{2}} \Delta t-\frac{1}{6} \frac{\partial^{3} q}{\partial t^{3}} \Delta t^{2} \\
& +\frac{(1-\theta)}{2} \frac{\Delta x}{\Delta t} \frac{\partial^{2} q}{\partial x^{2}} \Delta x-g h_{f} \frac{1}{24} \frac{\partial^{3} y}{\partial x^{3}} \Delta x^{2} .
\end{aligned}
$$

[35] This is very similar to the one obtained for the q-upwind scheme but without the unwanted zero-th order term that appears with that scheme. The diffusive term (third term on the RHS) is again of first order and proportional to $(1-\theta)$. The fact that diffusion can be adjusted is an important feature of the q-centered method which enables one to select the "right" amount of diffusion, preventing excessively smeared solutions (which typically result from the Lax scheme).

\section{Model Testing and Results}

[36] The numerical schemes proposed in sections 3.2 and 3.3 were coded within the LISFLOOD-FD hydraulic model of Bates and De Roo [2000]. LISFLOOD-FP has undergone extensive development since conception from a simple storage cell model to its current formulation based on the full and simplified forms of the Saint-Venant equations [e.g., Villanueva and Wright, 2006; Hunter et al., 2008;
Neal et al., 2009, 2011b; Bates et al., 2010]. The ability of this latest version to predict flood propagation has been extensively tested and compared with the results of other numerical models and analytical solutions of idealized scenarios [e.g., Bates et al., 2010; Neal et al., [2011a], as well as field data [Neal et al. [2011b].

[37] In this section the stability improvement introduced by the proposed numerical schemes is assessed against a structured sequence of numerical experiments that provide a rigorous test of its performance. Namely, the results of the q-upwind and q-centered schemes are compared with those of the Bates et al. [2010] formulation as well as with analytical solutions of the full-dynamic system. Moreover, results of a full-dynamic shallow water model implemented as part of the LISFLOOD-FD package are presented. This model solves the coupled 2-D system of hyperbolic PDEs using a first order finite volume (FV) Godunov method, with fluxes computed with the approximate Roe Riemann solver [Villanueva and Wright, 2006]. Only relatively low friction values (Manning's up to $n=0.035$ ) are tested here, as previous works have demonstrated the stability of the model at higher friction values. Specifically, the tests used here are: a nonbreaking wave propagation over a horizontal plane, a nonbreaking wave runup on a planar beach, and a simulation of flood propagation through a complex street and building network at fine spatial resolution.

\subsection{Test 1: Nonbreaking Wave Propagation Over a Horizontal Plane}

[38] The first numerical experiment consists of a wave propagation over a horizontal plane $(\partial z / \partial x=0)$ that is initially dry. This problem admits, for a particular set of boundary conditions, a solution of the full-dynamic shallow water equations, which provides an accurate benchmark for the assessment of the model's performance. This solution and the corresponding boundary condition were proposed by Hunter et al. [2005] by assuming a constant velocity distribution over the direction of wave propagation. Only a brief description of this solution is provided here. Rewriting the 1-D version of the shallow water equations as a function of velocity and depth,

$$
\begin{gathered}
\frac{\partial h}{\partial t}+\frac{\partial u h}{\partial x}=0 \\
\frac{\partial u}{\partial t}+u \frac{\partial u}{\partial x}+g\left(\frac{\partial h}{\partial x}+\frac{\partial z}{\partial x}\right)+g \frac{n^{2} u^{2}}{h^{4 / 3}}=0
\end{gathered}
$$

and imposing a constant velocity results:

$$
\begin{gathered}
\frac{\partial h}{\partial t}+u \frac{\partial h}{\partial x}=0, \\
\frac{\partial h}{\partial x}=-\left(\frac{\partial z}{\partial x}+\frac{n^{2} u^{2}}{h^{4 / 3}}\right) .
\end{gathered}
$$

[39] Equation (46) shows that the above assumptions lead to a solution in which the flow depth is simply advected at the constant flow velocity $u$ :

$$
h(x, t)=h(x-u t, 0)
$$


[40] Equation (47) is an ordinary differential equation which can be integrated analytically in the horizontal bed case, yielding:

$$
h=\left[-\frac{7}{3}\left(n^{2} u^{2} x+C\right)\right]^{3 / 7} .
$$

[41] In equation (49), $C$ is a constant of integration that can be obtained by imposing the moving boundary condition $h(u t, t)=0$, resulting in:

$$
h(x, t)=\left[-\frac{7}{3}\left(n^{2} u^{2}\{x-u t\}\right)\right]^{3 / 7} .
$$

[42] The upstream boundary condition is simply the solution of (50) at $x=0$ :

$$
h(0, t)=\left(\frac{7}{3} n^{2} u^{3} t\right)^{3 / 7}
$$

[43] The computational domain used in this simulation is composed of $32 \times 240$ square cells with $\Delta x=\Delta y=25 \mathrm{~m}$. The boundary condition spans over one of the sides of the domain, reducing the problem to a 1-D wave propagation. Two tests were run using values of Manning's coefficient

(a) $\mathrm{n}=0.01$

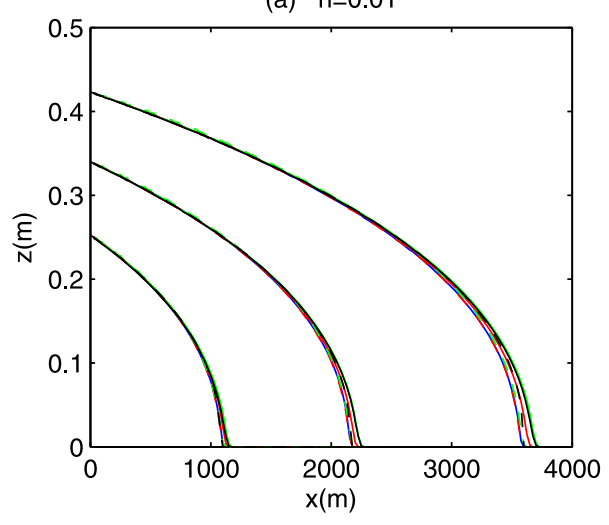

(c)

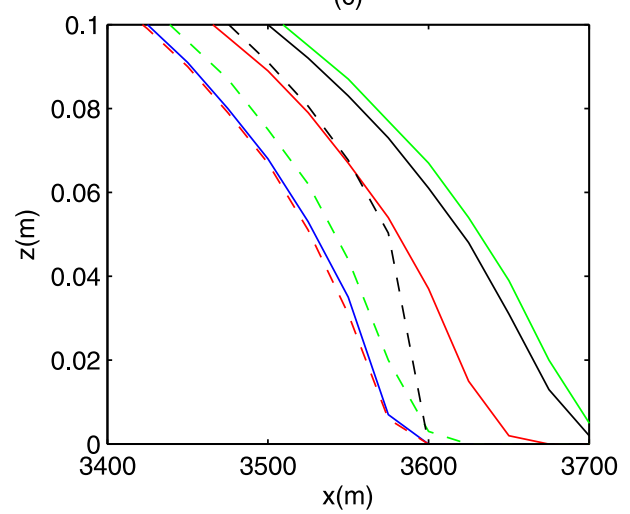

$\begin{array}{lll}\text { Semi- } \\ \text { implicit }\end{array} \quad \begin{aligned} & \text { upwind } \\ & \theta=0.9\end{aligned} \quad \begin{aligned} & \text { upwind } \\ & \theta=0.8\end{aligned}--\begin{aligned} & \text { centred } \\ & \theta=0.9\end{aligned}---\begin{aligned} & \text { centred } \\ & \theta=0.8\end{aligned}-\ldots$ Analytical - Roe

Figure 2. Top: predicted water surface elevation at $t=2700,5400$, and $9000 \mathrm{~s}$ using a uniform Manning coefficient of (a and c) 0.01 and (b and d) 0.005. Bottom: zoom-in of the wave fronts at $t=9000 \mathrm{~s}$.

of $n=0.01 \mathrm{sm}^{-1 / 3}$ and $n=0.005 \mathrm{sm}^{-1 / 3}$, and velocities of $u=0.4 \mathrm{~ms}^{-1}$ and $u=0.635 \mathrm{~ms}^{-1}$, respectively. These particular combinations of velocity and friction produce exactly the same boundary conditions in (51). The first friction value approximately represents the minimum surface roughness found in real-world applications (e.g., finished cement), while the second is used here only to demonstrate some characteristics of the numerical schemes.

[44] The time step during the simulations with the three inertial formulations gradually decreases as a result of the increasing water depths, reaching minimum values of $8.6 \mathrm{~s}$. The minimum time steps obtained in the simulation with the FV scheme are slightly shorter $(7.2$ and $6.6 \mathrm{~s}$, for $n=0.01$ and $n=0.03$, respectively).

[45] Figure 2 shows the water surface elevations predicted by each of the numerical schemes at different instants of the wave propagation for $n=0.01$ ( $2 \mathrm{a}$ and 2c) and $n=0.005$ ( $2 \mathrm{~b}$ and $2 \mathrm{~d}$ ), along with the corresponding analytical solutions. The figure illustrates important practical implications of the numerical schemes proposed. For $n=0.01$, the q-centered scheme propagates the wave at approximately the same speed predicted by the scheme of Bates et al. [2010] (e.g., Figures 2a and 2c). The wave speeds of both the q-centered and q-upwind schemes also compare reasonably well with the analytical solution, the former being slightly slower and the latter faster than this analytical solution at the wavefront. The q-centered

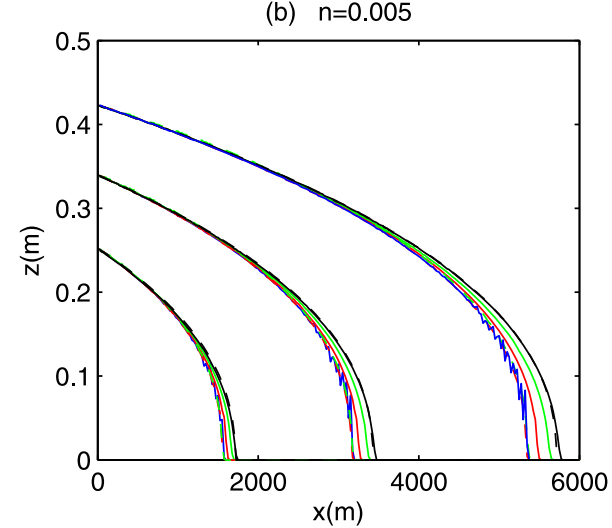

(d)

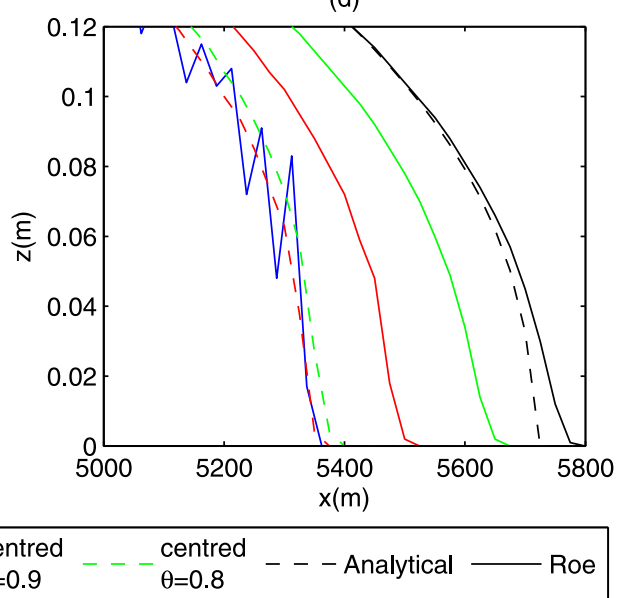


solutions with $\theta=0.8$ and 0.9 are very close, while the q-upwind results display a more significant dependence on $\theta$. The FV scheme propagates the wavefront slightly faster than the analytical solution, as would be expected from the numerical diffusion introduced by the first order scheme. No numerical oscillations were observed in these experiments with $n=0.01$.

[46] In the case of the even more severe experiment (i.e., $n=0.005$, Figures $2 \mathrm{~b}$ and $2 \mathrm{~d}$ ), small oscillations are observed in the solution obtained by the semi-implicit method, as previously reported by Bates et al. [2010]. The stabilizing effect of the numerical diffusion on the solution is evident in this example, with both the q-centered and q-upwind schemes providing solutions free from spurious oscillations, even when relatively high values of $\theta$ are used.

[47] Figures $2 \mathrm{~b}$ and $2 \mathrm{~d}$ show a nonnegligible discrepancy between position of the wavefronts predicted by the three inertial schemes and that corresponding to the analytical solution. This error is up to $6.2 \%$ for the q-centered and semi-implicit schemes and smaller in the case of the q-upwind. The wave speed predicted by the q-upwind method again displays a considerable dependency on the values of the parameter $\theta$.

[48] Differences in the wave propagation speeds observed in Figures $2 \mathrm{~b}$ and $2 \mathrm{~d}$ are highly influenced by elimination of the advective term in equation (4). This is because the analytical solution used here is a solution to the full-dynamic system, whereas the numerical solutions (i.e., semi-implicit, q-upwind, and q-centered) attempt to approximate the simplified inertial model. This key difference can be better understood in light of the differences in the characteristic speeds of the simplified inertial model (i.e., equations (12) and (13)) and those corresponding to the full-dynamic model:

$$
\lambda_{F D}=u \pm \sqrt{g h} .
$$

[49] Comparing (52) and (13) it is clear that right-going characteristic propagates the information faster in the fulldynamic system than in the simplified inertial, and this difference will depend on the relative magnitude of the first and second terms on the RHS of (52). When $u$ is small (i.e., relative to $\sqrt{g h}$ ), both models are expected to produce similar results, but they will generally deviate with an increase of $u$. In fact, this relative magnitude is directly related to the Froude number $F r=u / \sqrt{g h}$, so that higher characteristic speed differences will occur at higher Fr. This is what is observed in Figure 2. The velocity $u$ used in the $n=0.005$ test is $58 \%$ higher than in the $n=0.01$ test, while the boundary condition is held unchanged (i.e., the $\sqrt{g h}$ term is the same for both runs at the boundary). This represents a significant increase in $F r$, which leads to the wave speed discrepancies observed in Figure 2d. This effect of advection on the results is confirmed by the better agreement between the full-dynamic FV model and the analytical solution. The FV model solution also exhibits less diffusion at $n=0.005$ than at $n=0.01$ as a result of the milder water surface profile.

[50] The differences observed in Figure $2 \mathrm{~d}$ are thus inherently associated with the simplified nature of equations (3) and (4) (which neglect the convective acceleration term), and are expected to be higher at high $F r$. It must be emphasized, however, that the nonnegligible differences in the wave propagation speeds observed in Figure $2 \mathrm{~d}$ are highly influenced by the idealized nature of this test case, where an unrealistically smooth surface is used, and a constant velocity profile is assumed in order to obtain the analytical solution. In this test case the constant velocity assumption produces a flow with significantly high $\mathrm{Fr}$ in shallow zones of the domain. In fact, this leads to $\mathrm{Fr}$ tending to infinity as the depth approaches zero.

[51] The close agreement between the q-centered and semi-implicit schemes in stable experiments is important, as this previous version of the model has been the object of extensive tests involving a wide range of flow conditions in both idealized and real-world situations [e.g., Neal et al., 2011a, 2011b].

[52] On the other hand, the closer agreement between the q-upwind method and the analytical solution in the previous results should not be regarded as an advantage of this method. This is because the higher wave speed obtained is a result of the zero-th order term previously described, which obviously does not represent the physics of the advective term neglected in the simple inertial formulation. As previously demonstrated, the q-upwind method is inconsistent with the original system of equations (3) and (4), as the zero-th order term does not vanish with grid refinement.

[53] Numerical oscillations observed in Figure 2 are relatively small. This is a consequence of the specific boundary condition used to obtain the analytical solution. This particular slow variation in the upstream water depth produces a relatively smooth water surface profile. Only in the wavefront is this profile steep enough to develop oscillations in the solution, as can be observed in Figure 2d.

\subsection{Test 2: Nonbreaking Wave Runup on a Planar Beach}

[54] The second test case proposed by Hunter et al. [2005] explores the propagation of a wave over a planar beach (i.e., nonzero adverse longitudinal slope). This is a more stringent situation than that of the previous test case, as the additional decrease in the water depth caused by the adverse bed slope accentuates the nonlinear effects, enhancing the development of shocks.

[55] Equations (46) and (47) are still valid for this test case, and even though the analytical integration of (47) is not possible in the case of a nonhorizontal bed, an accurate solution can be obtained by numerical integration. Here the fourth order Runge-Kutta method was used to derive the benchmark solutions of the full-dynamic system and the corresponding boundary conditions used in the model.

[56] Two tests were run using values of Manning's coefficient of $n=0.03 \mathrm{sm}^{-1 / 3}$ and $n=0.01 \mathrm{sm}^{-1 / 3}$ and the same flow velocity $u=0.4 \mathrm{~ms}^{-1}$. The computational domain is again composed of $32 \times 240$ square cells with $\Delta x=\Delta y=25 \mathrm{~m}$, and an adverse slope value of $10^{-3}$ is used. The nonlinear variation in the boundary condition (variable water level), which also spans over one of the sides of the domain, is obtained by numerical integration.

[57] The minimum time steps obtained during the simulations with the three inertial formulations were 4.2 and $4.5 \mathrm{~s}$ for $n=0.03$ and $n=0.01$, respectively. In the simulation with the semi-implicit scheme and $n=0.01$, the model 
further reduced the time step (minimum value of $3.05 \mathrm{~s}$ ) as a result of numerical oscillations described in the following paragraphs. The FV model used minimum time steps of 3.7 and $4.1 \mathrm{~s}$ for $n=0.03$ and $n=0.01$, respectively.

[58] Water surface elevations predicted using each of the formulations at different instants of the wave propagation are shown in Figure 3. The results for $n=0.03$ show a good agreement between the semi-implicit and q-centered schemes and the analytical solution (Figure 3a), with both numerical schemes exhibiting only a small underestimation of the speed of the wavefront. The q-upwind results show a higher dependency on the value of $\theta$. The apparent better agreement between the analytical solution and this scheme with $\theta=0.9$ is a spurious effect of the errors terms introduced by the discretization, rather than an advantage of the method, as previously remarked. Results obtained with the full-dynamic FV model show a high degree of diffusion at the wavefront, which is a consequence of the first order approximation adopted. This effect is particularly significant in this experiment as a result of the combination of the higher Manning's coefficient used and the adverse slope, both of which induce relatively high gradients of flow depths (thus, increasing numerical diffusion). No numerical oscillations were observed with $n=0.03$.

(a) $\mathrm{n}=0.03$

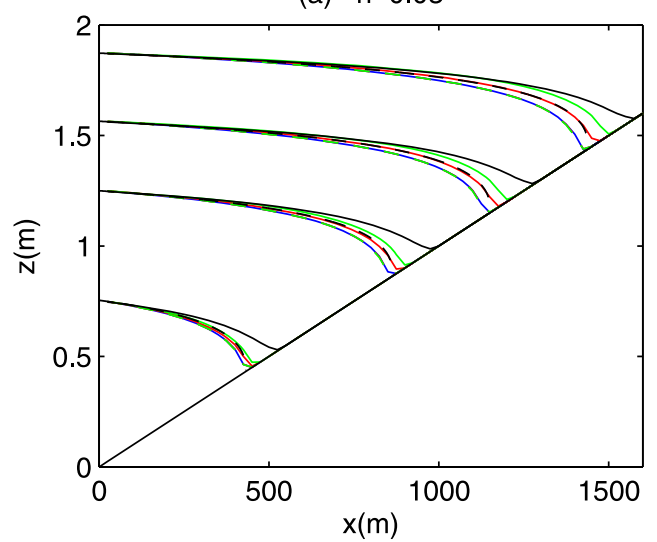

(c)

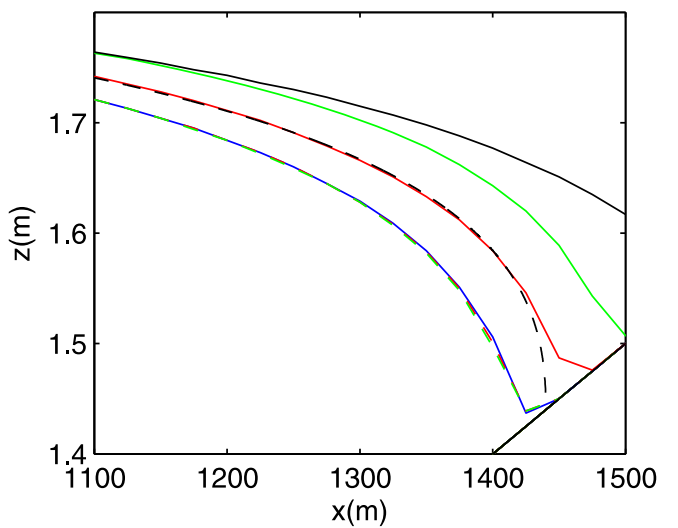

\begin{tabular}{|lll}
\hline Semi- \\
implicit
\end{tabular}$\quad \begin{aligned} & \text { upwind } \\
& \theta=0.9\end{aligned} \quad$\begin{tabular}{l} 
upwind \\
\hdashline$=0.8$
\end{tabular}$--\begin{aligned} & \text { centred } \\
& \theta=0.9\end{aligned}---\begin{aligned} & \text { centred } \\
& \theta=0.8\end{aligned}--\begin{aligned} & \text { Runge- } \\
& \text { Kutta }\end{aligned}-$ Roe

Figure 3. Top: predicted water surface elevation at $t=1080,2160,2880$, and $3600 \mathrm{~s}$ using a uniform Manning coefficient of ( $a$ and c) 0.03 and (b and d) 0.01. Bottom: zoom-in of the wave fronts at $t=3600 \mathrm{~s}$.
[59] The experiment with lower friction $(n=0.01$, Figures $3 \mathrm{~b}$ and $3 \mathrm{~d}$ ) demonstrates a remarkable improvement in the model stability introduced by the proposed numerical schemes, with smooth solutions obtained by both the q-centered and q-upwind methods, in contrast to the extreme oscillations observed in the solution of the semiimplicit scheme. These smooth solutions were obtained with relatively high values of $\theta$ (i.e., low levels of numerical diffusion). The speed of the wave propagated by the numerical schemes display the same characteristics observed in the previous examples, with the q-centered slightly slower, and the q-upwind showing a significant dependency on the values of $\theta$. The q-upwind scheme using $\theta=0.8$ particularly overestimates the position of the wavefront. Results using lower values of $\theta$ (not shown here) showed a further increase in the gap between the q-upwind results and the analytical solution, and even adverse water surface profiles predicted in the wavefront. This clearly reflects the effect of the zero-th order error term in equation (38), which is proportional to $\theta$. The FV model also produced satisfactory results, although the wavefront is highly diffused, for the reasons previously discussed.

[60] Both the results of these simulations and the analysis of the modified equations indicate that the q-centered

(b) $\mathrm{n}=0.01$

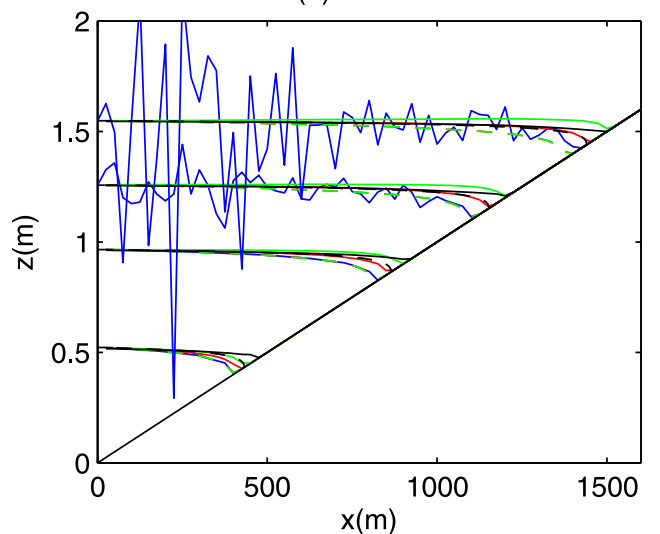

(d)

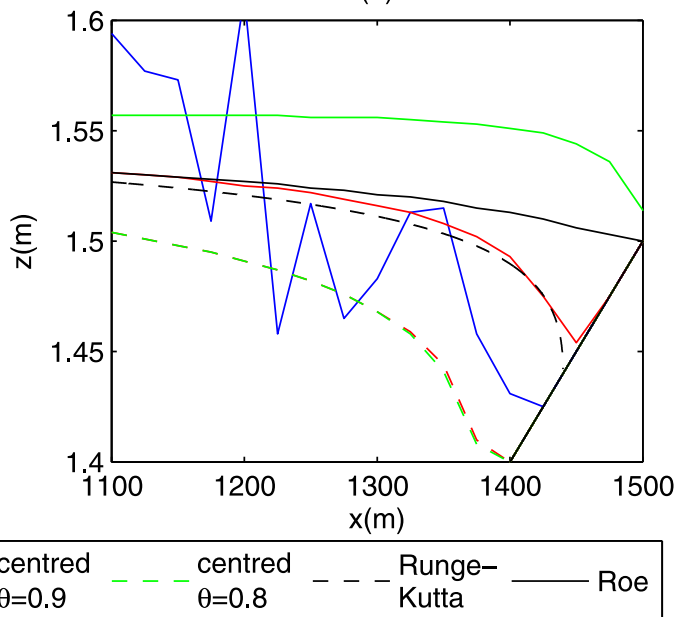


scheme provides the best solution for the simplified inertial system of equations (i.e., equations (3) and (4)).

\subsection{Test 3: Fine Spatial Resolution Simulation of Urban Inundation}

[61] In sections 4.1 and 4.2 we have analyzed the performance of the three numerical schemes studied in this paper in terms of numerical stability and accuracy using idealized numerical experiments. Results have shown some important advantages introduced by the q-centered scheme. In this section we further examine the model modifications proposed in this paper by testing its ability to simulate flood propagation over a complex topography. To this end, we used both the semi-implicit and q-centered schemes to simulate a flood episode over a $4.6 \times 2.5 \mathrm{~km}$ domain in a highly urbanized area of North Greenwich, London.

[62] A digital elevation model (DEM) at $2 \mathrm{~m}$ resolution (i.e., $\Delta x=\Delta y=2 \mathrm{~m}$ ) provided by the Environment Agency of England and Wales (EA) was used in this simulation. This DEM was generated from an airborne laser altimetry survey (conducted in March 1999), which has been filtered to remove vegetation and building features to leave a "bare earth" digital terrain model (DTM). Furthermore, a significant amount of manual processing has been carried out by the EA to remove bridges and elevated road sections that would otherwise form artificial blockages to flood propagation [Fewtrell, 2008]. The final DTM has the vertical accuracy of approximately $5 \mathrm{~cm}$ root-mean-square error. A high-accuracy digital map layer (Ordnance Survey Mastermap ${ }^{\circledR}$ ) was then used to reintroduce buildings back into the DTM. This was carried out by setting the height of all buildings to $15 \mathrm{~m}$ above the DTM elevation, which is much higher than the maximum water elevation predicted in the simulations, thus preventing water from flowing over the top of the buildings. Figure 4 shows the final DEM used in the simulation overlaid on the aerial photography of the area. The site contains a mix of high-density housing, light industrial zones, dockyard areas, road and rail links, and major urban infrastructure such as the $\mathrm{O} 2$ arena and the Blackwall road tunnel which runs from within the domain underneath the River Thames.

[63] The inflow boundary condition used in this simulation consists of a number of unsteady discharge point sources along the north and west sides of the computational

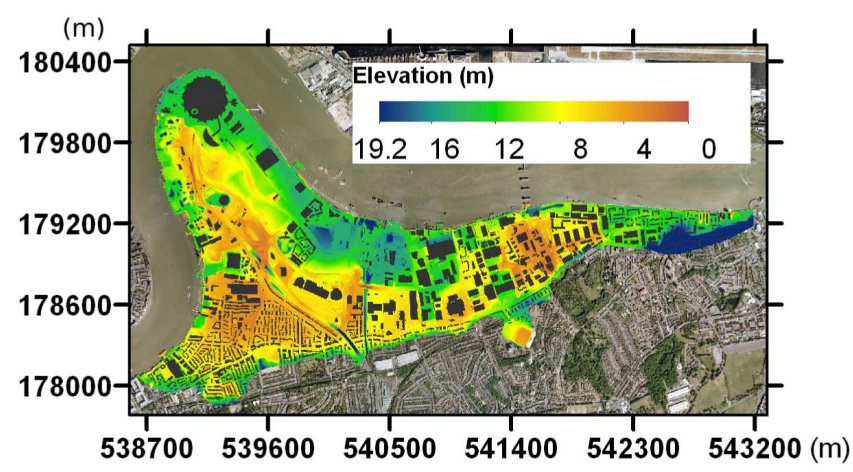

Figure 4. Digital elevation model (DEM) overlayed with the aerial photography of North Greenwich, London. Black areas represent buildings defined from the Ordnance Survey Mastermap ${ }^{\circledR}$ layer. domain which are used to represent the water flowing over the top of the river Thames' levees. These individual hydrographs were obtained from a study conducted by HR Wallingford Ltd. using a model which integrates a full range of loading conditions (water levels) with the performance and integrity of flood defenses (Gouldby et al. [2007], extracted from Fewtrell [2008]; Gouldby et al. [2008]). The specific scenario presented here corresponds to a 200 -yr return period event combined with the failure of two defenses.

[64] The simulations were carried out with both the q-centered $(\theta=0.9)$ and the semi-implicit schemes, and using spatially uniform values of the Manning coefficient of $n=0.035$ and $n=0.025$. These Manning's values correspond to surface roughness realistically found in urban areas and were chosen here to illustrate both the onset of oscillations in the solution of the semi-implicit method as well as the improvements obtained with the q-centered scheme in a real-world situation. The minimum time step obtained during these simulations was $0.17 \mathrm{~s}$.

[65] Figure 5 presents the water depths predicted by both schemes at $t=1620 \mathrm{~s}$ and $t=7200 \mathrm{~s}$ using $n=0.035$, as well as maps of absolute differences in water depths (semiimplicit minus q-centered). These results show a general good agreement between the two formulations, with differences limited to $\pm 5 \mathrm{~cm}$ over most of the domain. Only in a few isolated zones do the predictions differ by more than $20 \mathrm{~cm}$.

[66] When the friction is reduced (i.e., $n=0.025$, Figure 6) the differences between the two models are significantly increased. In Figure 6c, for example, the area of the domain where differences exceed $\pm 5 \mathrm{~cm}$ is substantially greater than the corresponding area in Figure 5c. Furthermore, some regions of the domain exhibit a marked alternation of water level values at neighboring cells, indicating the presence of oscillations in the solution (see Figure 7 for a zoom-in of Figure 6f). In order to visualize these numerical oscillations, water surface profiles were extracted from a 180-m long section within the domain (see Figure 7 for the exact location) at $t=1620 \mathrm{~s}$ (Figure 8). Results for $n=0.035$ show only minor differences between the two formulations and very small oscillations in the solution of the semi-implicit model. However, these oscillations are considerably increased when the Manning coefficient is reduced $(n=0.025)$, while the new formulation (i.e., q-centered scheme) still delivers a smooth solution.

[67] It is important to emphasize that these improvements are not merely a cosmetic exercise, as the oscillations observed in Figure 8 can introduce additional problems to the model, and surfaces with Manning's friction values as low as $n=0.01-0.015$ occur commonly in urban areas [Chow, 1959]. In particular, in shallow regions of the domain these oscillations can lead to negative values of the water depth. The current implementation of the model handles this unphysical situation by resetting the negative values to zero so that the model can advance to the next time step. This is equivalent to artificially adding water into the domain, which can lead to important mass balance problems. In fact, comparing the mass errors (difference between the net inflow through the boundaries and the change in the water volume inside the domain, integrated over time) for each simulation provides a valuable insight 
(a) Semi-implicit $\mathrm{t}=1620 \mathrm{~s}$

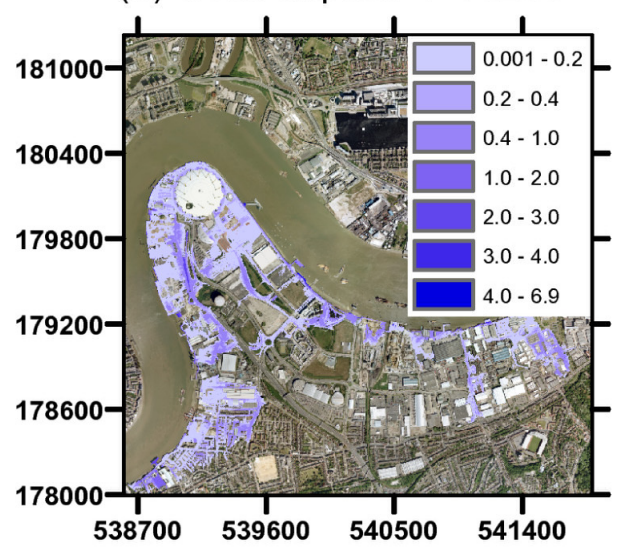

(b) q-centred $\mathrm{t}=1620 \mathrm{~s}$

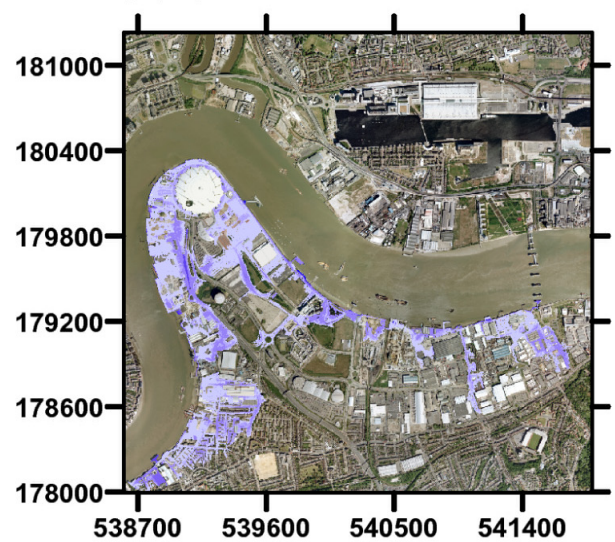

(c) Semi-implicit - $\mathrm{q}$-centred $\mathrm{t}=1620 \mathrm{~s}$

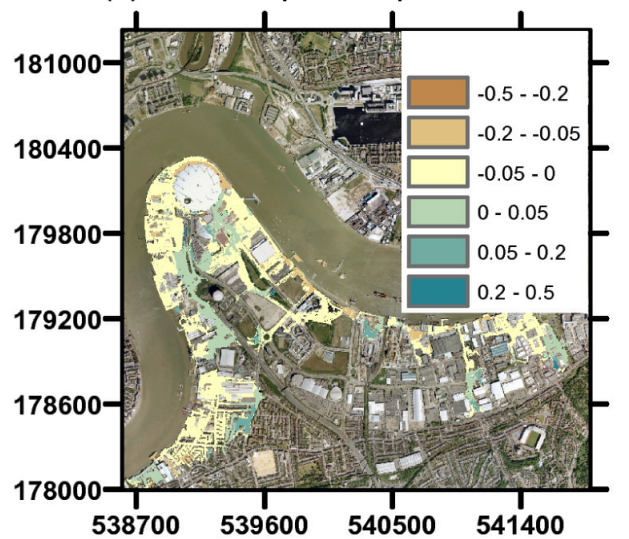

(d) Semi-implicit $t=7200 \mathrm{~s}$

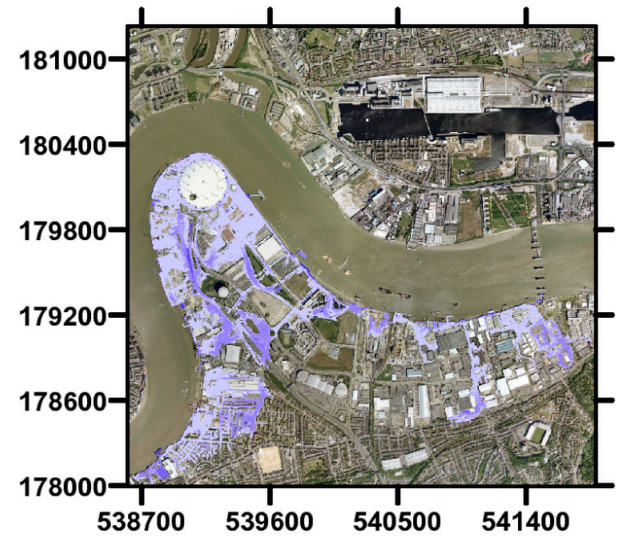

(e) q-centred $t=7200 \mathrm{~s}$

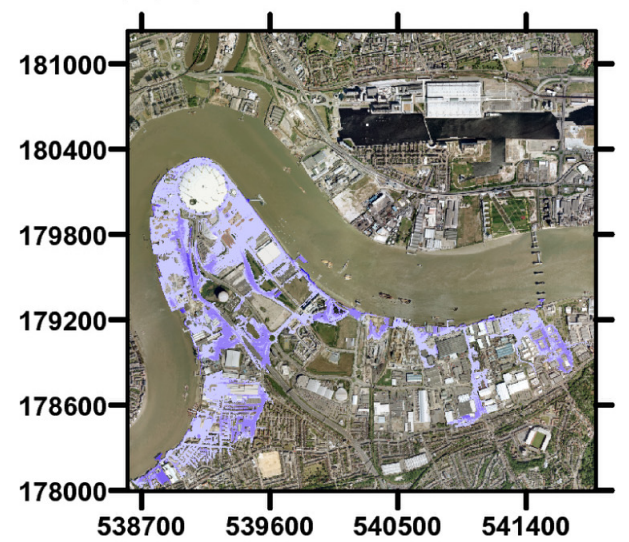

$\begin{array}{llll}538700 & 539600 & 540500 & 541400\end{array}$

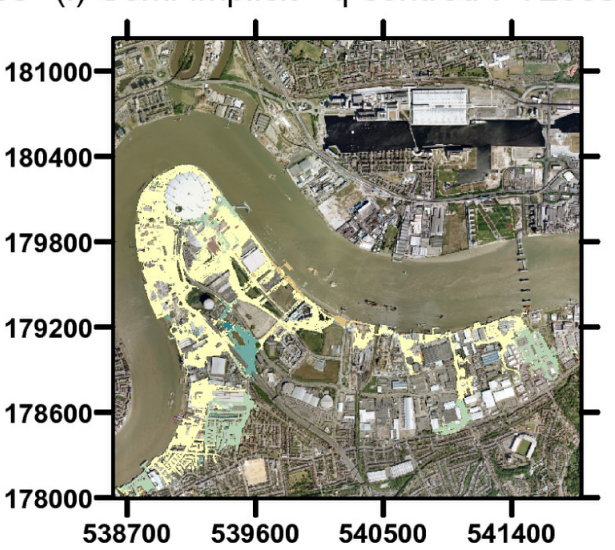

Figure 5. Water depths predicted by (a and d) the semi-implicit and (b and e) the q-centered formulations, as well as (c and f) maps of absolute differences (semi-implicit minus q-centered). Results at (left) $t=1620 \mathrm{~s}$ and (right) $t=7200 \mathrm{~s}$ obtained using $n=0.035$. All units are in meters.

into the behavior of the solution. In the previous tests the mass errors obtained over the whole simulation by both formulations with $n=0.035$ are of the same order of magnitude and smaller than $1 \mathrm{~m}^{3}$ (i.e., smaller than $0.0005 \%$ of the total volume of water in the domain at the end of the simulation, $V_{T}=2.20 \times 10^{5} \mathrm{~m}^{3}$ ). On the other hand, the simulations carried out using $n=0.025$ introduced mass errors of more than $3 \times 10^{3} \mathrm{~m}^{3}$ for the semi-implicit method and only $9.6 \mathrm{~m}^{3}$ with the new q-centered formulation.

[68] The stability improvements introduced by the q-centered scheme add a very small computational cost to the model compared to the semi-implicit formulation. The computational time (CPU time using a single thread implementation and a Dual-Core AMD Opteron ${ }^{\circledR}$ Processor 2218) for the simulation with both $n=0.035$ and $n=0.025$ 
(a) Semi-implicit $\mathrm{t}=1620 \mathrm{~s}$

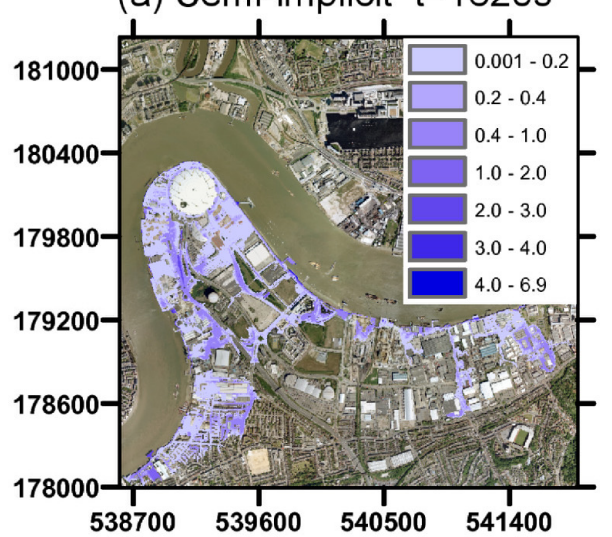

(b) q-centred $\mathrm{t}=1620 \mathrm{~s}$

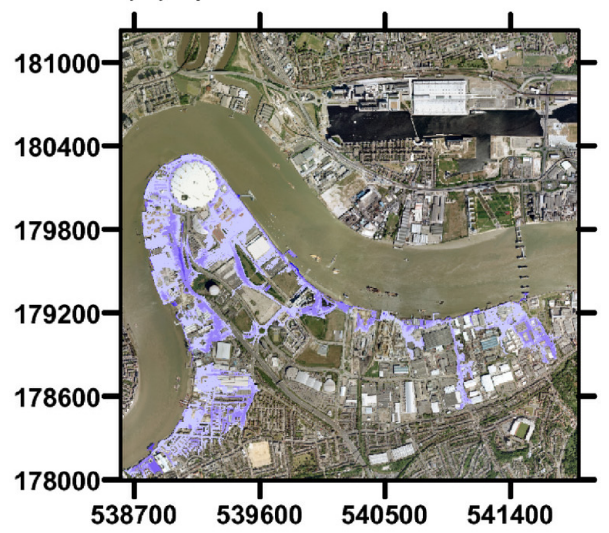

(c) Semi-implicit - qcentred $\mathrm{t}=1620 \mathrm{~s}$

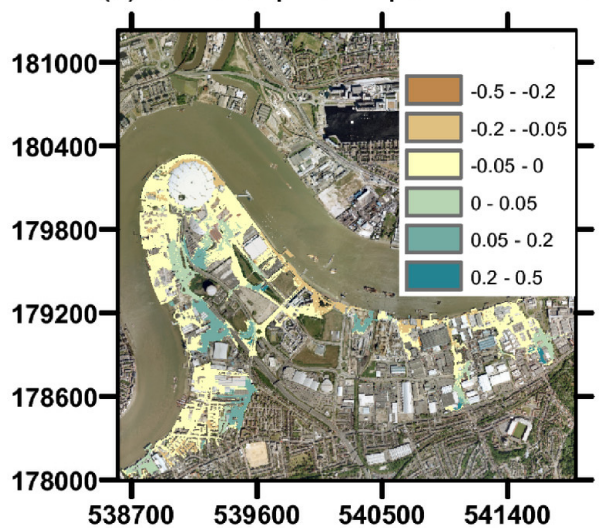

(d) Semi-implicit $t=7200 \mathrm{~s}$

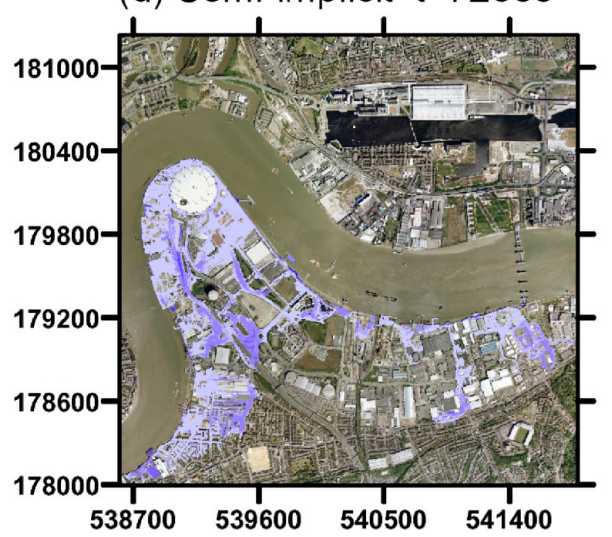

(e) q-centred $t=7200 \mathrm{~s}$

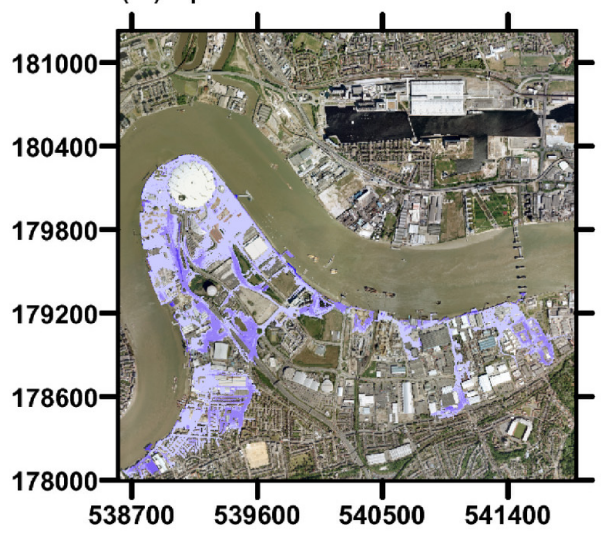

(f) Semi-implicit - -centred $t=7200$ s

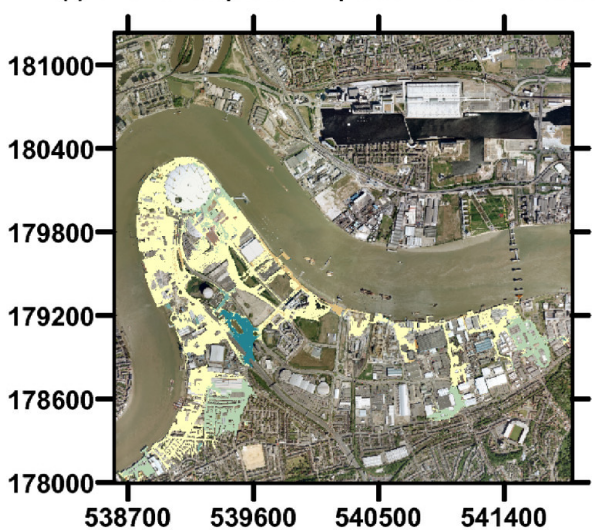

Figure 6. Water depths predicted by (a and d) the semi-implicit and (b and e) the q-centered formulations, as well as (c and f) maps of absolute differences (semi-implicit minus q-centered). Results at (left) $t=1620 \mathrm{~s}$ and (right) $t=7200 \mathrm{~s}$ obtained using $n=0.025$. All units are in meters.

were $223 \mathrm{~min}$ with the semi-implicit scheme and $232 \mathrm{~min}$ with the q-centered method, representing an approximately $4 \%$ increase in time. On the other hand, the enhanced stability provided by the q-centered scheme enables longer time steps to be used, which more than offset this additional computational cost. As an example, the same simulations were carried out with the value of $\alpha=0.8$ in equation (15). Results show that the q-centered scheme preserves the solution smoothness under this increased time step and the differences between water depths computed with both values of $\alpha$ are negligible (e.g., at $t=1620 \mathrm{~s}$ these differences are greater than $1 \mathrm{~cm}$ in only $3 \%$ of the wet cells). However, the computation time of these simulations (i.e., using the q-centered scheme with $\theta=0.9$ and $\alpha=0.8$ ) were 203 and $204 \min$ for $n=0.035$ and $n=0.025$, respectively. A similar reduction in the computational time was observed for the semi-implicit method with $\alpha=0.8$ (196 min) but now larger oscillations were observed for $n=0.035$. In the case of $n=0.025$ these oscillations were so high that the simulation with the semi-implicit method had to be 


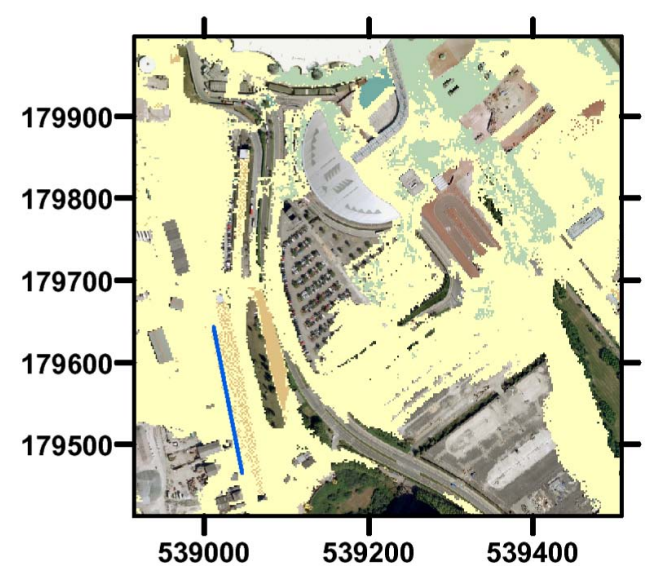

Figure 7. Zoom-in of Figure 6f. The blue line corresponds to the cross section presented in Figure 8. All units are in meters.

manually aborted. This example therefore illustrates the fact that not only does the proposed method improve the model stability, but furthermore it can be used to speed up computations as a result of the possibility of using longer time steps.

\section{Conclusions}

[69] Previous attempts to solve a simplified inertial formulation for 2-D shallow water problems [Bates et al., 2010] have reported substantial stability issues in the case of low friction scenarios. In this paper we first provided further mathematical insights into this simplified system of equations and the corresponding finite difference method proposed by Bates et al. [2010]. A system of modified equations was derived which unveiled the lack of numerical diffusive terms in this previous numerical scheme. As the stabilizing effect of friction is reduced, the nondiffusive scheme becomes unable to counteract the destabilizing effect of discontinuities developing in the solution as a result of the nonlinear characteristic of the system, ultimately leading to oscillations in the solution.

[70] Two numerical schemes, namely the "q-upwind" and "q-centered" formulations, were proposed, analyzed,

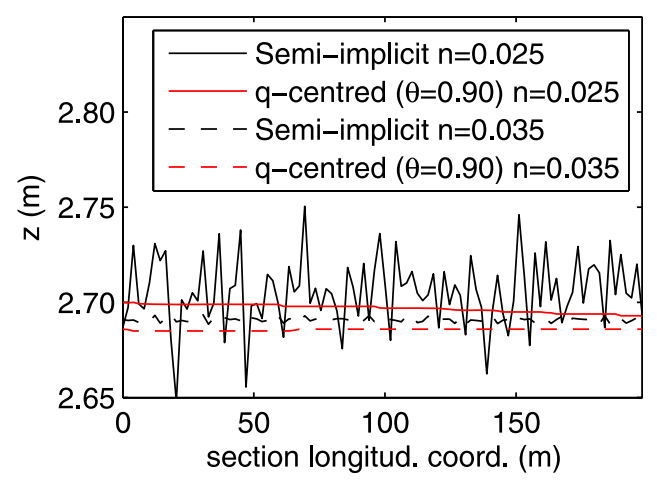

Figure 8. Water surface elevation profiles extracted from a $180-\mathrm{m}$ long section (see Figure 7 for the exact location). Results obtained at $t=1620 \mathrm{~s}$ using the semi-implicit and q-centered $(\theta=0.9)$ schemes for $n=0.025$ and $n=0.035$. and tested in idealized and real-world scenarios. The modified equations derived for the proposed schemes showed an additional numerical diffusive term that considerably stabilizes the solution. Analysis of the same equations also exposed an unwanted zero-th order term in the case of the q-upwind method, which does not vanish with grid refinement. Simulations using both schemes confirmed the effect of numerical diffusion in stabilizing the solution. Furthermore, results showed a very good agreement between the predictions obtained with the q-centered scheme and the scheme proposed by Bates et al. [2010], as well as with analytical solutions available for two idealized test cases. The new scheme propagates the wave at the same speed as the Bates et al. [2010] method, although some oscillations appear in the latter, while the former delivers a smooth solution even at extremely low friction conditions. On the other hand, results obtained with the q-upwind method showed a significant dependency on the values of the parameter $\theta$ as a result of the zero-th order error term. Results under extremely (unrealistically) low friction have also exposed a general limitation of local inertial formulations in predicting wave propagation problems at relatively high Froude numbers.

[71] The q-centered scheme uses a discretization similar to the Lax diffusive method, but here three points are used (instead of two points used by the Lax method) to approximate the value of the variable at the beginning of the time step. One of the features of the proposed method is that the degree of diffusivity can be adjusted in order to stabilize the solution while preventing the development of excessively smeared solutions.

[72] The stability improvements proposed in this paper were introduced at a negligible additional computational cost. Moreover, the enhanced stability of the q-centered scheme allows the use of longer time steps, which more than counterbalance this additional cost. Results from a real-world simulation have demonstrated that this trade-off can actually result in an increase in the overall computational efficiency of the model. This robust, yet computationally efficient formulation, paves the way to a range of applications that hitherto have been hindered by the lack of stability at low friction scenarios. This is particularly true for whole city risk analysis at very high resolution, where the presence of smooth areas in the urban domain imposed an important limitation for the previous formulation.

[73] Acknowledgments. The authors wish to thank the Environment Agency of England and Wales for provision of the LiDAR data for this project. The research was funded under UK Natural Environment Research Council grant NE/I005366/1 awarded to the DEMON project ("Developing Enhanced impact MOdels for integration with Next generation NWP and climate outputs").

\section{References}

Arico, C., M. Sinagra, L. Begnudelli, and T. Tucciarelli (2011), MAST-2D diffusive model for flood prediction on domains with triangular Delaunay unstructured meshes, Adv. Water Resour., 34, 1427-1449, doi:10.1016/ j.advwatres.2011.08.002.

Aronica, G., T. Tucciarelli, and C. Nasello (1998), 2D Multilevel model for flood wave propagation in flood-affected areas, J. Water Resour. Plann. Manage., 124, 210-217, doi:10.1061/(ASCE)0733-9496(1998) 124:4(210).

Bates, P. D., and A. P. J. De Roo (2000), A simple raster-based model for flood inundation simulation, J. Hydrol., 236, 54-77, doi:10.1016/S00221694(00)00278-X. 
Bates, P. D., M. S. Horritt, and T. J. Fewtrell (2010), A simple inertial formulation of the shallow water equations for efficient two-dimensional flood inundation modelling, J. Hydrol., 387, 33-45, doi:10.1016/ j.jhydrol.2010.03.027.

Bermudez, A., C. Rodriguez, and M. A. Vilar (1991), Solving shallow water equations by a mixed implicit finite element method, IMA J. Numer. Anal., 11, 79-97.

Bradford, S. F., and B. F. Sanders (2002), Finite-volume model for shallowwater flooding of arbitrary topography, J. Hydraul. Eng., 128, 289-298, doi:10.1061/(ASCE)0733-9429(2002)128:3(289).

Bradford, S. F., and B. F. Sanders (2005), Performance of high-resolution, nonlevel bed, shallow-water models, J. Hydraul. Eng., 131, 1073-1081, doi:10.1061/(ASCE)0733-9399(2005)131:10(1073).

Brown, J. D., T. Spencer, and I. Moeller (2007), Modeling storm surge flooding of an urban area with particular reference to modeling uncertainties: A case study of Canvey Island, United Kingdom, Water Resour. Res., 43, W06402, doi:10.1029/2005WR004597.

Chow, V. T. (1959), Open-Channel Hydraulics, 680 pp., McGraw-Hill, N. Y.

Cunge, J. A., F. M. Holly, and A. Verwey (1980), Practical Aspects of Computational River Hydraulics, 420 pp., Pitman, London, U.K.

Fewtrell, T. J. (2008), Development of simple methods for two-dimensional hydraulic models of urban flooding, $\mathrm{PhD}$. dissertation submitted to the University of Bristol, Bristol, U.K.

Fewtrell, T., P. D. Bates, M. Horritt, and N. Hunter (2008), Evaluating the effect of scale in flood inundation modelling in urban environments, Hydrol. Processes, 22, 5107-5118, doi:10.1002/hyp.

Gouldby, B., P. Sayers, O. Tarrant, and D. Kavanagh (2007), Thames Estuary 2100: Performance based asset management, Tech. Rep. IA8/10, HR Wallingford, UK.

Gouldby, B., P. Sayers, J. Mulet-Marti, M. A. A. M. Hassan, and D. Benwell (2008), A methodology for regional-scale flood risk assessment, Proceedings of the Institution of Civil Engineers, Water Management, 161, pp. 169-182, doi:10.1680/wama.2008.161.3.169.

Guinot, V., and S. Frazão (2006), Flux and source term discretization in two-dimensional shallow water models with porosity on unstructured grids, Int. J. Numer. Method Fluids, 50, 309-345.

Hervouet, J. M. (2000), TELEMAC modelling system: An overview, Hydrol. Processes, 14, 2209-2210, doi:10.1002/1099-1085(200009) $14: 13<2209$ : :AID-HYP23>3.3.CO;2-Y.

Hervouet, J. M., and A. Petitjean (1999), Malpasset dam break revisited with two-dimensional computations, J. Hydraul. Res., 37(6), 777-788.

Horritt, M. S., and P. D. Bates (2001), Effects of spatial resolution on a raster based model of flood flow, J. Hydrol., 253, 239-249.

Hunter, N. M., M. S. Horritt, P. D. Bates, M. D. Wilson, and M. G. F. Werner (2005), An adaptive time step solution for raster-based storage cell modelling of floodplain inundation, Adv. Water Resour., 28, 975991, doi:10.1016/j.advwatres.2005.03.007.

Hunter, N. M., P. D. Bates, M. S. Horritt, and M. D. Wilson (2006), Improved simulation of flood flows using storage cell models, Proceedings of the Institution of Civil Engineers, Water Manage., 159(1), 9-18.
Hunter, N. M., et al. (2008), Benchmarking 2D hydraulic models for urban flooding, Water Manage., 161, 13-30, doi:10.1680/wama.2008.161.1.13.

Lax, P., and B. Wendroff (1960), Systems of conservation laws, Commun. Pure Appl. Math., 13(12), 217-237.

LeVeque, R. J. (2002), Finite Volume Methods for Hyperbolic Problems, 257 pp., Cambridge Univ. Press, Cambridge, Mass.

LeVeque, R. J., and D. L. George (2008), High-resolution finite volume methods for the shallow water equations with bathymetry and dry states, in Advanced Numerical Models for Simulating Tsunami Waves and Runup, Advances in Coastal and Ocean Engineering, vol. 10, edited by P. L. Liu, C. Synolakis, and H. Yeh, pp. 43-73, World Scientific, Singapore, available at http://www.amath.washington.edu/rj1/pubs/catalina04/.

Li, S., and C. J. Duffy (2011), Fully coupled approach to modeling shallow water flow, sediment transport, and bed evolution in rivers, Water Resour. Res., 47, W03508, doi:10.1029/2010WR009751.

Neal, J., I. Villanueva, N. Wright, T. Willis, T. Fewtrell, and P. Bates (2011a), How much physical complexity is needed to model flood inundation, Hydrol. Processes, doi:10.1002/hyp.8339, in press.

Neal, J., G. Schumann, T. Fewtrell, M. Budimir, P. Bates, and D. Mason (2011b), Evaluating a new LISFLOOD-FP formulation with data from the summer 2007 floods in Tewkesbury, UK, J. Flood Risk Manage., 4 , 85-95, doi:10.1111/j.1753-318X.2011.01093.x.

Neal, J. C., P. D. Bates, T. J. Fewtrell, N. M. Hunter, M. D. Wilson, and M. S Horritt (2009), Distributed whole city water level measurements from Carlisle 2005 urban flood event and comparison with hydraulic model simulations, J. Hydrol., 368, 42-55, doi:10.1016/j.jhydrol.2009.01.026.

Peraire, J., O. C. Zienkiewicz, and K. Morgan (1986), Shallow water problems: A general explicit formulation, Int. J. Num. Methods Eng., 22, 547-574.

Ponce, V. M. (1990), Generalized diffusion wave equation with inertial effects, Water Resour. Res., 26(5), 1099-1101.

Ponce, V. M., R. M. Li, and D. B. Simons (1978), Applicability of kinematic and diffusion models, J. Hydraul. Eng., 104, 353-360.

Sanders, B., J. E. Schubert, and H. A. Gallegos (2008), Integral formulation of shallow-water equations with anisotropic porosity for urban flood modeling, J. Hydrol., 362, 19-38.

Toro, E. F. (2001), Shock-capturing methods for free-surface shallow flows, John Wiley, Chichester, U. K.

Toro, E. F., and P. Garcia-Navarro (2007), Godunov-type methods for freesurface shallow flows: A review, J. Hydraul. Res., 45(6), 736-751.

van Leer, B. (1979), Towards the ultimate conservative difference scheme. V. A second-order sequel to Godunov's method, J. Comput. Phys., 32, 101-136.

Villanueva, I., and N. G. Wright (2006), Linking Riemann and storage cell models for flood prediction, Proceedings of the Institution of Civil Engineers, Water Management, 159, 27-33.

Xia, R. (1994), Impact of coefficients in momentum equation on selection of inertial models, J. Hydraul. Res., 32, 615-621.

P. Bates, G. A. M. de Almeida, J. E. Freer, and M. Souvignet, School of Geographical Sciences, University of Bristol, University Rd., Bristol BS8 1SS, UK. (gustavo.dealmeida@bristol.ac.uk) 